$10,11,05$

\title{
Фазовые переходы и термодинамические свойства модели Поттса с числом состояний спина $q=4$ на решетке кагоме
}

\author{
(C) А.К. Муртазаев, Т.Р. Ризванова, М.К. Рамазанов, М.А. Магомедов \\ Институт фоизики ДФИЦ РАН, \\ Махачкала, Россия \\ E-mail: sheikh77@mail.ru
}

Поступила в Редакцию 28 февраля 2020 г.

В окончательной редакции 28 февраля 2020 г.

Принята к публикации 3 марта 2020 г.

На основе алгоритма Ванга-Ландау методом Монте-Карло выполнены исследования фазовых переходов и термодинамических свойств двумерной ферромагнитной модели Поттса с числом состояний спина $q=4$ на решетке кагоме. Используя метод кумулянтов Биндера четвертого порядка и гистограммный анализ данных проведен анализ характера фазовых переходов. Установлено, что в исследуемой модели наблюдается фазовый переход первого рода.

Ключевые слова: фазовые переходы, метод Монте-Карло, алгоритма Ванга-Ландау, модель Поттса, решетка кагоме.

DOI: $10.21883 /$ FTT.2020.08.49614.043

\section{1. Введение}

Исследование фазовых переходов (ФП), критических, магнитных и термодинамических свойств низкоразмерных решеточных моделей имеет большой научный интерес. Это связано с тем, что такие модели описывают большой класс реальных физических систем [1-5]. В частности, двумерные магнетики открывают широкие перспективы для практического применения. Использование таких материалов в индустрии наносистем может привести к большим успехам [6-8].

В настоящее время для исследования магнитных спиновых систем используют различные решеточные модели. Одними из наиболее часто используемых моделей для изучения ФП, критических и термодинамических свойств таких систем являются модели Изинга, $X Y$ и Гейзенберга. При исследовании двумерных и трехмерных систем на основе этих моделей получено много интересных результатов. Для модели Поттса существует совсем немного надежно установленных фактов. Модель Поттса, является теоретическим инструментом, применяемым для изучения широкого класса явлений и объектов в физике конденсированных сред. Несмотря на интенсивные теоретические исследования двумерных спиновых решеточных систем, описываемых моделями Поттса, в течение последних пятидесяти лет, к настоящему времени существует совсем немного надежно установленных фактов. На сегодняшний день достаточно хорошо изучена модель Поттса с числом состояний спина $q=2$ и $q=3$ на различных типах решеток и почти все ее свойства известны [9-17].

Двумерная модель Поттса с числом состояний спина $q=4$ до сих пор малоизучена. Данная модель интересна тем, что значение $q=4$ является граничным значением интервала $2 \leq q \leq 4$, где наблюдается ФП второго рода и области значений $q>4$, в котором ФП происходит как переход первого рода [18]. Согласно результатам работ $[4,19,20]$, для модели Поттса с числом состояний спина $q=2,3$ и 4 наблюдается ФП второго рода. Однако, при $q=4$ в рассматриваемой модели были обнаружены особенности термодинамического поведения. При исследовании модели Поттса с $q=4$ основное внимание до сих пор уделялось спиновым системам на треугольной [21] и гексагональной $[22,23]$ решетках.

Отдельный интерес представляет изучение ФП и термодинамических свойств модели Поттса с числом состояний спина $q=4$ на решетке кагоме. Интерес к этой модели обусловлен следующими основными причинами.

Во-первых, природа ФП и термодинамические свойства модели Поттса зависят от числа состояний спина $q$ и от геометрии решетки и этот вопрос все еще недостаточно полно изучен.

Во-вторых, модель Поттса на решетке кагоме обладает многообразием ее структурных свойств.

В-третьих, исследований ФП и термодинамических свойств модели Поттса с $q=4$ на решетке кагоме практически нет.

В связи с этим, в данной работе, нами предпринята попытка на основе метода Монте-Карло (МК) провести исследование ФП и термодинамических свойств двумерной ферромагнитной модели Поттса с числом состояний спина $q=4$ на решетке кагоме.

Из данных, полученных на сегодняшний день, нельзя однозначно определить характер ФП и закономерности изменения термодинамического поведения данной модели и эти вопросы до сих пор остаются открытыми. Исследование двумерной модели Поттса с $q=4$ на основе современных методов и идей позволит получить ответ на ряд вопросов, связанных с ФП и термодинамическими свойствами низкоразмерных решеточных систем. 


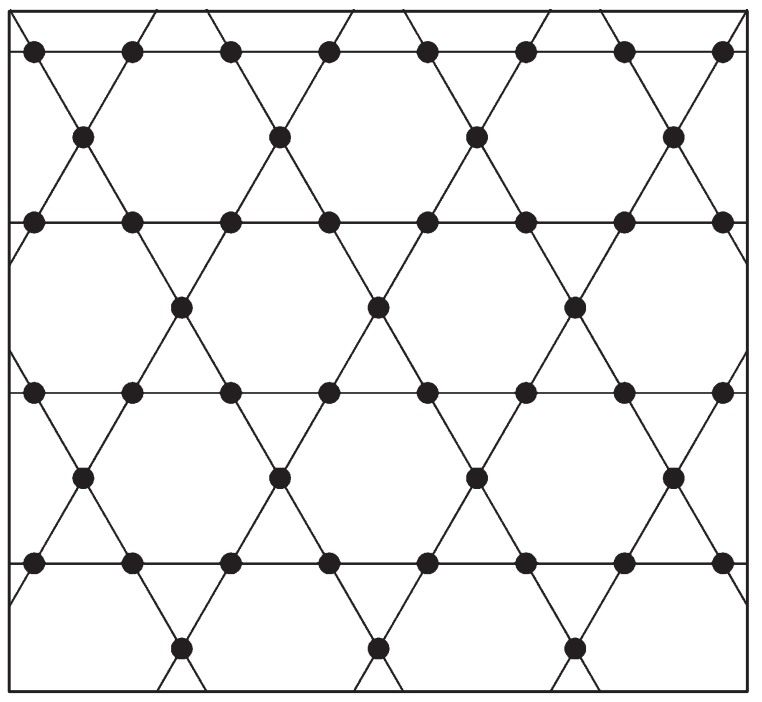

Рис. 1. Решетка кагоме.

\section{2. Модель и метод исследования}

Гамильтониан модели Поттса с числом состояний спина $q=4$ может быть представлен в следующем виде:

$$
H=-J \sum_{i, j} \cos \theta_{i, j}
$$

где $J$ - параметр обменного ферромагнитного взаимодействия для ближайших соседей, $\theta_{i, j}-$ угол между взаимодействующими спинами $S_{i}-S_{j}$. Схематически эта модель представлена на рис. 1. Данная модель может быть использована для описания различных соединений, имеющие квазидвумерную структуру: Капелласиты (Kapellasite, $\mathrm{Cu}_{3} \mathrm{Zn}(\mathrm{OH})_{6} \mathrm{Cl}_{2}$ ), Гербертсмититы $\left(\mathrm{ZnCu}_{3}(\mathrm{OH})_{6} \mathrm{Cl}_{2}\right)$, Яроситы и т.д.

В настоящее время такие системы на основе микроскопических гамильтонианов успешно изучаются на основе метода МК [24-28]. В последнее время разработано много новых вариантов алгоритмов метода МК. Одними из наиболее эффективных для исследования подобных систем является алгоритм Ванга-Ландау [29,30]. Поэтому нами, в данном исследовании был использован этот алгоритм.

Алгоритм Ванга-Ландау является реализацией метода энтропийного моделирования и позволяет вычислить функцию плотности состояний системы. Этот алгоритм основан на том, что совершая случайное блуждание в пространстве энергий с вероятностями, обратно пропорциональными плотности состояний $g(E)$ мы получаем равномерное распределение по энергиям. Подобрав вероятности перехода такими, что посещение всех энергетических состояний стало бы равномерным, можно получить изначально неизвестную плотность состояний $g(E)$, зная которую можно вычислить значения необходимых термодинамических параметров при любой температуре. Так как плотность состояний $g(E)$ очень быстро растет с увеличением размеров исследуемых систем, для удобства хранения и обработки больших чисел пользуются величиной $\ln g(E)$.

Алгоритм Ванга-Ландау был использован нами в следующем виде.

Задается произвольная начальная конфигурация спинов. Стартовые значения плотности состояний $g(E)=1$, гистограммы распределений по энергиям $H(E)=0$, стартовый модификационный фактор $f=f_{0}=e^{1}$ $\approx 2.71828$. Многократно совершаем шаги в фазовом пространстве, пока не получим относительно плоскую гистограмму $H(E)$ (т.е. пока не будут посещены примерно одинаковое количество раз все возможные энергетические состояния системы). При этом вероятность перехода из состояния с энергией $E_{1}$ в состояние с энергией $E_{2}$ определяется по формуле $p=g\left(E_{1}\right) / g\left(E_{2}\right)$. Если переход в состояние с энергией $E_{2}$ состоялся, то $g\left(E_{2}\right) \rightarrow j \times g\left(E_{2}\right), H\left(E_{2}\right) \rightarrow H\left(E_{2}\right)+1$, иначе $g\left(E_{1}\right) \rightarrow f \times g\left(E_{1}\right)$. Если гистограмма стала „Плоской“ то обнуляем гистограмму $H(E) \rightarrow 0$, уменьшаем модификационный фактор $f \rightarrow \sqrt{f}$, и продолжаем снова, пока $f \geq f_{\text {min. }}$ В нашем случае $f_{\text {min }}=1.0000000001$. Таким образом, определив плотность состояний системы, можно рассчитать значения термодинамических параметров при любой температуре. В частности, внутреннюю энергию $U$, свободную энергию $F$, теплоемкость $C$ и энтропию $S$ можно вычислить, используя следующие выражения:

$$
\begin{gathered}
U(T)=\frac{\sum_{E} E g(E) e^{-E / k_{B} T}}{\sum_{E} g(E) e^{-E / k_{B} T}} \equiv\langle E\rangle_{T}, \\
F(T)=-k_{B} T \ln \left(\sum_{E} g(E) e^{-E / k_{B} T}\right), \\
C=\left(N K^{2}\right)\left(\left\langle U^{2}\right\rangle-\langle U\rangle^{2}\right), \\
S(T)=\frac{U(T)-F(T)}{T},
\end{gathered}
$$

где $K=|J| / k_{B} T, N-$ число частиц, $T-$ температура (здесь и далее температура дана в единицах $|J| / k_{B}$ ).

Для анализа характера ФП нами был использован метод кумулянтов Биндера четвертого порядка [31] и гистограммный метод анализа данных метода МК [24,25].

Расчеты проводились для систем с периодическими граничными условиями и линейными размерами $L \times L=N, L=24 \div 96$.

\section{3. Результаты моделирования}

На рис. 2 представлена плотность состояний $g(E)$ для систем с различными линейными размерами $L$ (здесь и далее статистическая погрешность не превышает размеров символ, использованных для построения зависимостей). Из рисунка видно, что плотность состояний $g(E)$ в основном состоянии для всех $L$ стремится к нулю. Такое поведение плотности состояний связано с отсутствием вырождения основного состояния системы. 


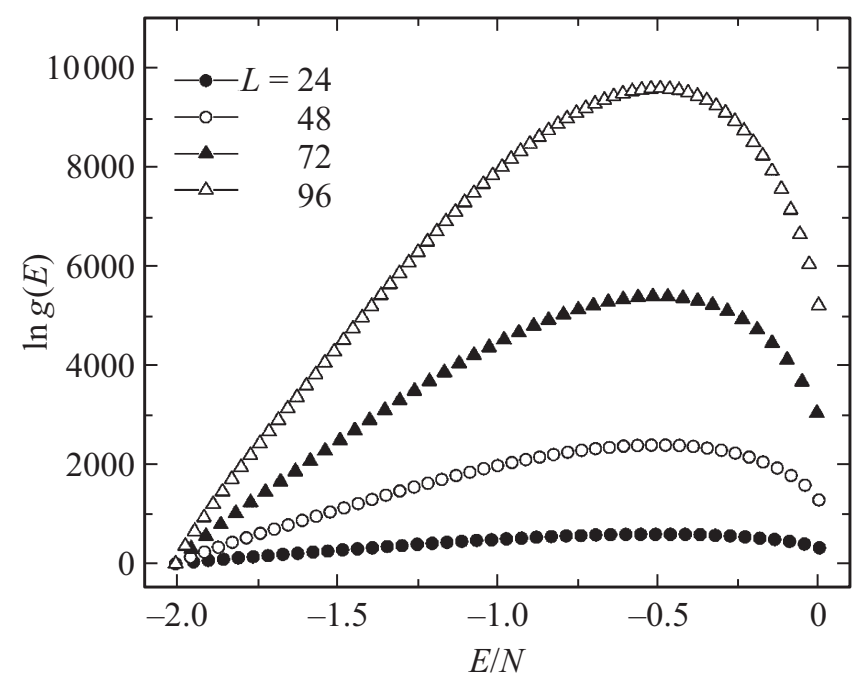

Рис. 2. Плотность состояний $g(E)$.

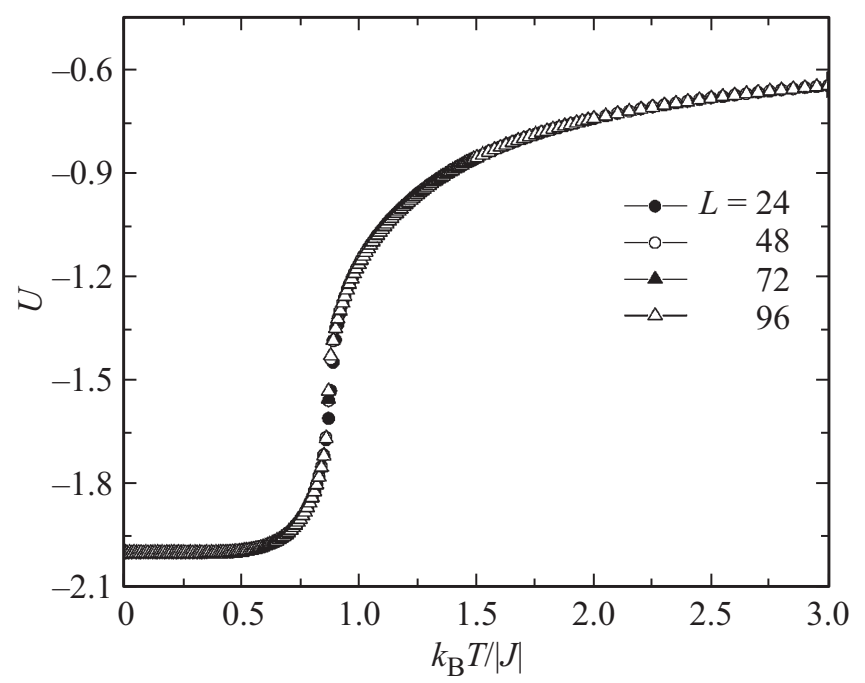

Рис. 3. Температурные зависимости внутренней энергии $U$.

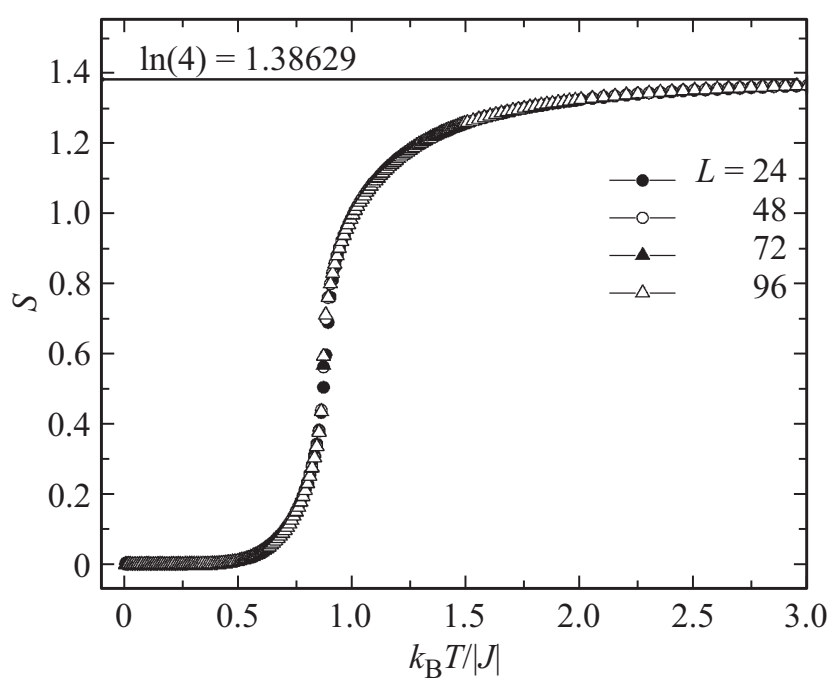

Рис. 4. Температурные зависимости энтропии $S$.

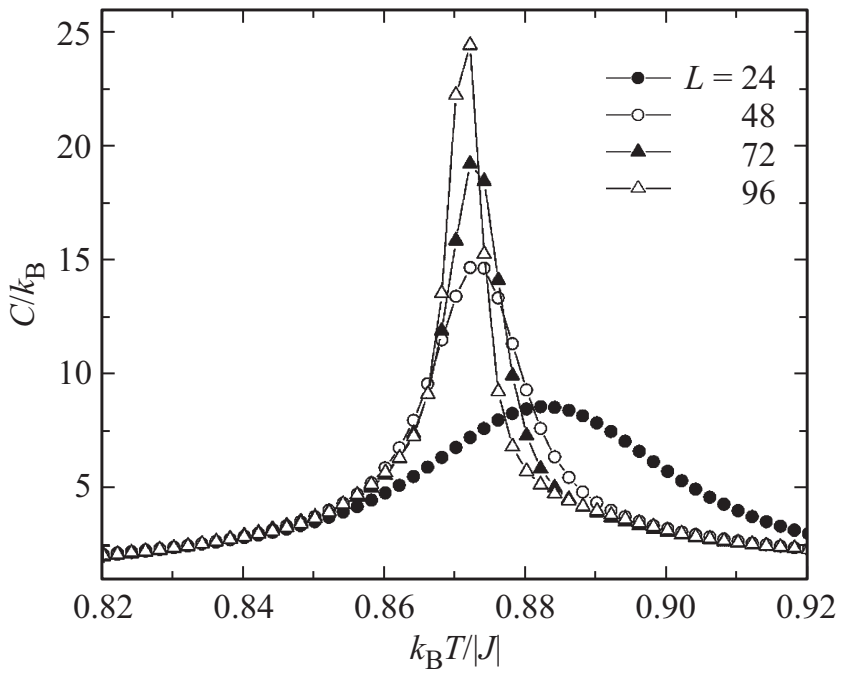

Рис. 5. Температурные зависимости теплоемкости $C / k_{B}$.

Температурные зависимости внутренней энергии $U$ для систем с различными линейными размерами представлены на рис. 3. На рисунке видно, что вблизи критической области для всех значений $L$ наблюдается резкий скачок, что характерно для ФП первого рода.

Температурные зависимости энтропии $S$ для систем с различными $L$ приведены на рис. 4 . На рисунке видно, что для всех рассмотренных значений $L$ с увеличением температуры энтропия системы стремится к теоретически предсказанному значению $\ln 4$. При низких температурах энтропия системы стремится к нулю. Такое поведение энтропии свидетельствует о том, что в данной модели вырождение основного состояния отсутствует.

На рис. 5 представлены характерные зависимости теплоемкости $C$ от температуры для систем с различными линейными размерами. Отметим, что в зависимости теплоемкости от температуры для всех систем вблизи критической температуры наблюдаются хорошо выраженные максимумы, которые увеличиваются с ростом числа спинов в системе, причем эти максимумы с увеличением значения $L$ в пределах погрешности приходятся на одну и ту же температуру. Это свидетельствует, во-первых, о высокой эффективности использованного способа добавления периодических граничных условий, а во-вторых, о достижении насышения по $N$ для многих исследуемых нами параметров.

Для анализа особенностей поведения тепловых характеристик вблизи критической точки и определения критической температуры $T_{C}$ наиболее эффективным является метод кумулянтов Биндера четвертого порядка [31]:

$$
\begin{aligned}
& V_{L}=1-\frac{\left\langle U^{4}\right\rangle_{L}}{3\left\langle U^{2}\right\rangle_{L}^{2}}, \\
& U_{L}=1-\frac{\left\langle m^{4}\right\rangle_{L}}{3\left\langle m^{2}\right\rangle_{L}^{2}},
\end{aligned}
$$

где $V_{L}$ - энергетический кумулянт, $U_{L}-$ магнитный кумулянт. 


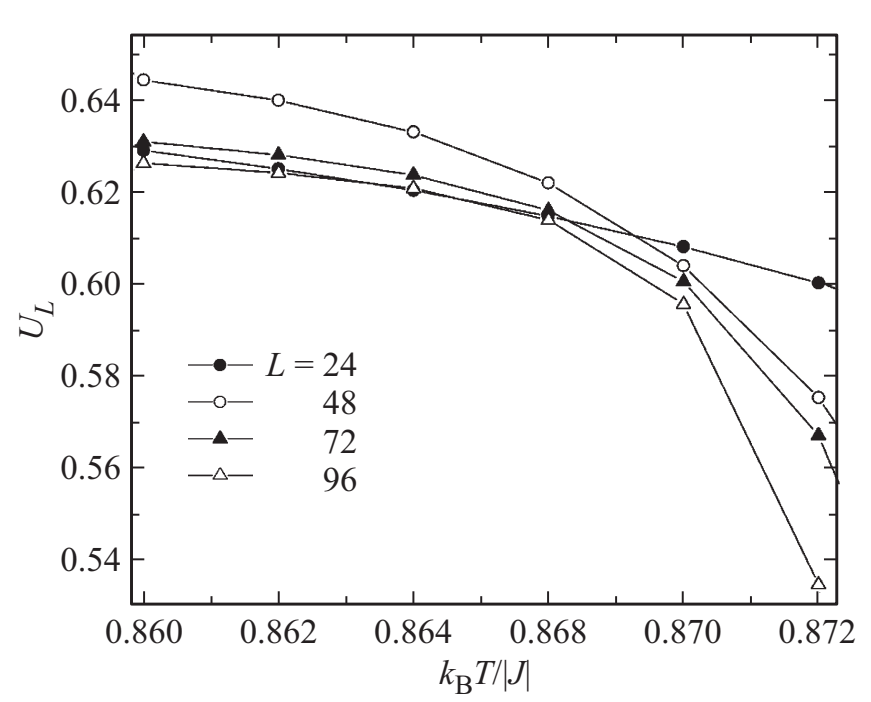

Рис. 6. Температурные зависимости магнитного кумулянта Биндера $U_{L}$.

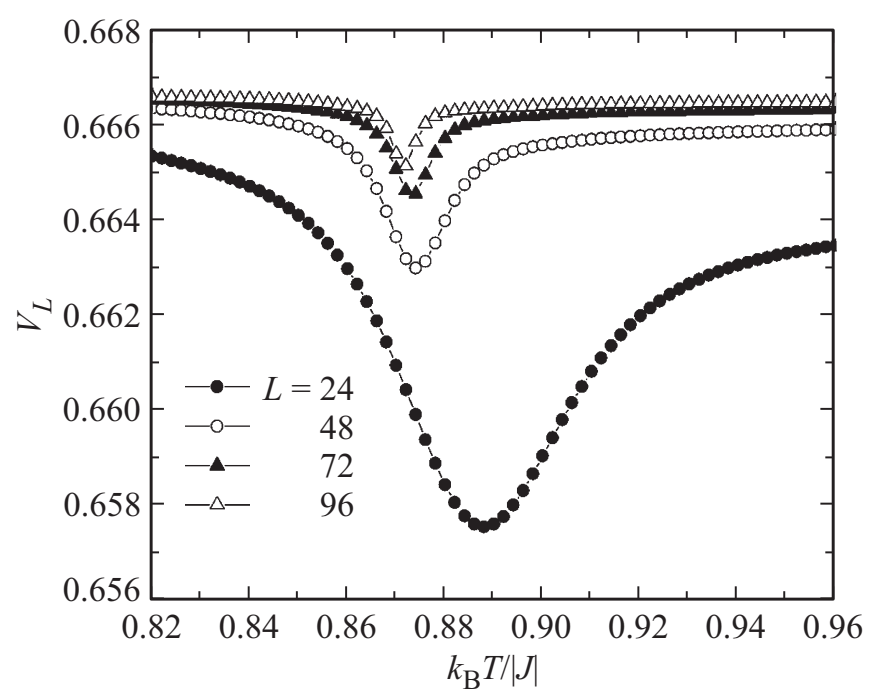

Рис. 7. Температурные зависимости энергетического кумулянта Биндера $V_{L}$.

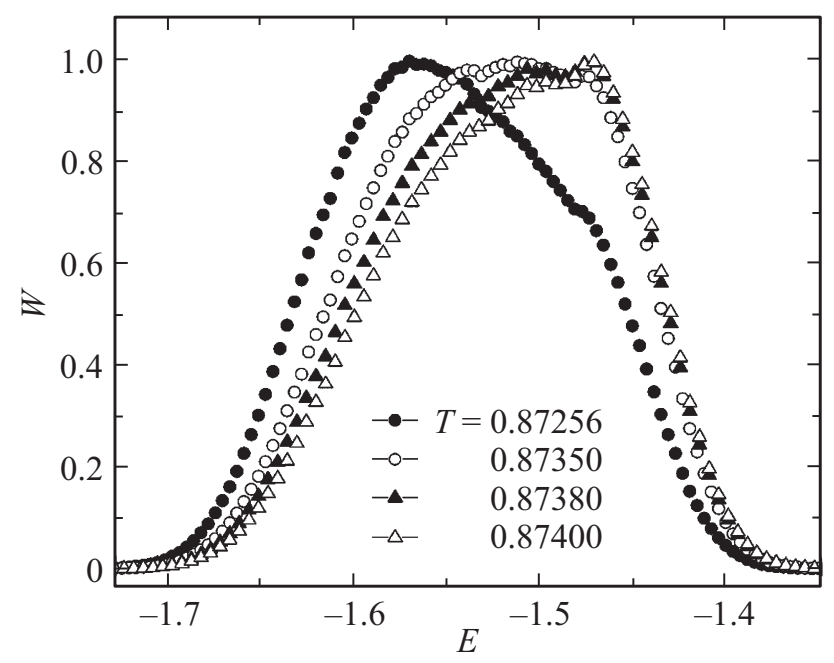

Рис. 8. Гистограммы распределения энергии для $L=96$ при различных температурах.
Выражения (6) и (7) позволяют определить критическую температуру $T_{C}$ с большой точностью для ФП первого и второго рода соответственно. Следует отметить, что применение кумулянтов Биндера позволяет также хорошо тестировать тип ФП в системе. Известно, что ФП первого рода характеризуются тем, что величина $V_{L}$ стремится к некоторому нетривиальному значению $V^{*}$ согласно выражению

$$
V_{L}=V^{*}+b L^{-d}
$$

при $L \rightarrow \infty$ и $T=T_{C}(L)$, где величина $V^{*}$ отлична от $2 / 3$, а минимальная величина $U_{L \min }\left(T=T_{\min }\right)$ расходится $U_{L \min }\left(T=T_{\min }\right) \rightarrow-\infty$ при $L \rightarrow \infty$.

В случае ФП второго рода кривые температурной зависимости кумулянтов Биндера $U_{L}$ имеют четко выраженную точку пересечения [31].

На рис. 6 представлена характерная зависимость $U_{L}$ от температуры для разных значений $L$. Из графика видно, что в критической области отсутствует четко выраженная точка пересечения, что свидетельствует в пользу наличия в системе ФП первого рода. Температурная зависимость энергетического кумулянта $V_{L}$ для разных значений $L$ представлена на рис. 7. Как видно из графика величина $V_{L}$ стремится к $2 / 3$, а величина $V^{*}=2 / 3$, что характерно для ФП второго рода. Эта величина рассчитана используя выражение (8).

Поэтому, для более подробного анализа рода ФП нами использовался гистограммный анализ данных метода МК. Этот метод позволяет надежно определить род ФП. Методика определения рода ФП этим методом подробно описана в работах $[24,25,32]$.

Результаты настоящей работы, полученные на основе гистограммного анализа, показывают, что ФП в данной модели является переходом первого рода. Это продемонстрировано на рис. 8. На этом рисунке представлены гистограммы распределения энергии для системы с линейными размерами $L=96$. Графики построены при различных температурах близких критической температуре. Из рисунка видно, что в зависимости вероятности $W$ от энергии $E$ вблизи критической температуры наблюдаются два максимума, которые свидетельствует в пользу ФП первого рода. Наличие двойного пика на гистограммах распределения энергии является достаточным условием для ФП первого рода. Как видно на рисунке, двойные пики наблюдаются в маленьком интервале температур $0.8735<T<0.874$. Ниже и выше указанного интервала один пик исчезает, что усложняет определение типа ФП в таких системах. Такое поведение характерно для систем, в которых происходят ФП первого рода близкие к переходам второго рода.

\section{4. Заключение}

Исследование фазовых переходов и термодинамических свойств двумерной ферромагнитной модели Поттса с числом состояний спина $q=4$ на решетке кагоме 
выполнено с использованием алгоритма Ванга-Ландау метода Монте-Карло. На основе гистограммного метода и метода кумулянтов Биндера проведен анализ характера фазовых переходов. Показано, что в системе наблюдается фазовый переход первого рода.

\section{Финансирование работы}

Исследование выполнено при финансовой поддержке Российского фонда фундаментальных исследований в рамках научного проекта № 19-02-00153-а.

\section{Конфликт интересов}

Авторы заявляют, что у них нет конфликта интересов.

\section{Список литературы}

[1] H.T. Diep. Frustrated Spin Systems, World Scientific Publishing Co. Pte. Ltd., Singapore (2004).

[2] Р. Бэкстер. Точно решаемые модели в статистической механике. Мир, М. (1985).

[3] F.Y. Wu. Exactly Solved Models: A Journey in Statistical Mechanics. World Scientific, N. J. (2008).

[4] F.Y. Wu. Rev. Mod. Phys. 54, 235 (1982).

[5] W. Zhang, Y. Deng. Phys. Rev. E 78, 031103 (2008).

[6] A. Nakano, M.E. Bachlechner, R.K. Kalia, F. Lidorikis, P. Vashishta, G.Z. Voyiadjis, T.J. Campbell, S. Ogata, F. Shimojo. Comput. Sci. Eng. 3, 56 (2001)

[7] T. Fabritius, N. Laflorencie, S. Wessel. Phys. Rev. B 82, 035402 (2010).

[8] R. Masrour, L. Bahmad, A. Benyoussef. J. Magn. Magn. Mater. 324, 3991 (2012).

[9] N. Schreiber, R. Cohen, S. Haber. Phys. Rev. E 97, 032106 (2018).

[10] D.P. Foster, C. Gérard. Phys. Rev. B 70, 014411 (2004).

[11] I. Puha, H.T. Diep. J. Appl. Phys. 87, 5905 (2000).

[12] M. Nauenberg, D.J. Scalapino. Phys. Rev. Lett. 44, 837 (1980).

[13] J.L. Cardy, M. Nauenberg, D.J. Scalapino. Phys. Rev. B 22, 2560 (1980).

[14] M.K. Ramazanov, A.K. Murtazaev, M.A. Magomedov. Physica A 521, 543 (2019).

[15] S. Ostlund. Phys. Rev. B 24, 398 (1981).

[16] M. Quartin, S.L.A. de Queiroz. J. Phys. A 36, 951 (2003).

[17] D.P. Foster, C. Gérard. J. Phys. A 35, L75 (2002).

[18] H. Feldmann, A.J. Guttmann, I. Jensen, R. Shrock, S.-H. Tsai. J. Phys. (A 31), 2287 (1998).

[19] M. Nauenberg, D.J. Scalapino. Phys. Rev. Lett. 44, 837 (1980).

[20] J.L. Cardy, M. Nauenberg, D.J. Scalapino. Phys. Rev. B 22, 2560 (1980).

[21] А.К. Муртазаев, М.К. Рамазанов, Д.Р. Курбанова. ФТТ 61, 2195 (2019).

[22] А.К. Муртазаев, М.К. Рамазанов, М.К. Мазагаева, М.А. Магомедов. ЖЭТФ 156, 502 (2019).

[23] М.К. Рамазанов, А.К. Муртазаев, М.А. Магомедов, М.К. Мазагаева. ФТТ 62, 442 (2020).

[24] М.К. Рамазанов, А.К. Муртазаев. Письма в ЖЭТФ 103, 522 (2016).

[25] М.К. Рамазанов, А.К. Муртазаев. Письма в ЖЭТФ 106, 72 (2017).
[26] А.К. Муртазаев, М.А. Магомедов, М.К. Рамазанов. Письма в ЖЭТФ 107, 265 (2018).

[27] М.К. Рамазанов, А.К. Муртазаев. Письма в ЖЭТФ 109, 610 (2019).

[28] А.К. Муртазаев, М.К. Рамазанов, К.Ш. Муртазаев, М.А. Магомедов, М.К. Бадиев. ФТТ 62, 229 (2020).

[29] F. Wang, D.P. Landau. Phys. Rev. E 64, 056101 (2001).

[30] F. Wang, D.P. Landau. Phys. Rev. Lett. 86, 2050 (2001).

[31] K. Binder, D.W. Heermann. Monte Carlo Simulation in Statistical Physics. Springer-Verlag, Berlin (1988); Nauka, Moscow (1995). P. 214.

[32] А.К. Муртазаев, Д.Р. Курбанова, М.К. Рамазанов. ЖЭТФ 156, 980 (2019).

Редактор Д.В. Жуманов 